\title{
Temperature spectrum of the solar wind turbulence
}

\author{
Gogoberidze G. ${ }^{* 1}$, Machabeli G. ${ }^{\dagger 1}$, and Voitenko Yu. ${ }^{\ddagger 2}$ \\ ${ }^{1}$ Ilia State University, 3/5 Cholokashvili ave., 0162 Tbilisi, Georgia \\ ${ }^{2}$ Belgian Institute for Space Aeronomy, Ringlaan-3-Avenue Circulaire, B-1180 Brussels, Belgium
}

\begin{abstract}
We show that there exists apparent contradiction between the temperature spectra derived from the Spektr- $R$ data and the temperature spectra predicted theoretically. We show that the temperature fluctuations can be correctly estimated from the Spektr- $R$ data only if the mean temperature is isotropic. Since the mean temperature in the solar wind is usually anisotropic, the derived fluctuations appear to be pseudo-temperature rather than temperature. These pseudotemperature fluctuations are driven by the high-amplitude magnetic fluctuations in Alfvén waves rather than the fluctuations of temperature or thermal velocity.
\end{abstract}

Keywords: Solar Wind: Turbulence

\section{Introduction}

Plasma ion moments (density, velocity and temperature) in the solar wind are usually derived using in-situ data collected by means of Faraday cups onboard a spacecraft. Although Faraday cups are quite simple devices, derivation of the plasma moments based on the currents measured by the Faraday cups is nontrivial and requires some kind of nonlinear fitting technique and some assumptions about particle distribution functions. Because of the complex fitting procedures, determination of the measurement errors in the obtained plasma moments is also nontrivial (Bruno \& Carbone, 2013, Gogoberidze et al., 2012b, Hnat et al., 2011, Kasper et al., 2002).

Recently, Safrankova et al. (2013) analyzed spectra of velocity, density and thermal speed in the frequency range $0.001-2 \mathrm{~Hz}$, therefore covering both magnetohydrodynamic (MHD) and kinetic ranges. These spectra were obtained using measurements of the Bright Monitor of the Solar Wind on board the Spektr-R spacecraft. The authors found that the spectral indices and spectral breaks between MHD and kinetic ranges were very similar for the bulk velocity and thermal speed, whereas the spectral behavior of the density fluctuations was entirely different. We found these results surprising because velocity perturbations are mostly due to the dominant Alfvénic component of the turbulence (Gogoberidze et al., 2012a), whereas the density and temperature fluctuations belong to the subdominant compressible fraction. This means it is natural to expect similar behavior of the temperature and density spectra rather than temperature and velocity.

In the present paper we attempt to understand this contradiction by analyzing dynamics of high frequency perturbations in the solar wind and methods of their measurements by Spektr-R. We show that some plasma parameters derived from the Faraday cup data can be strongly affected by the anisotropy of the proton distribution function. In particular, the derived thermal velocity is strongly dominated by perturbations of the magnetic field (and not parallel and/or perpendicular proton temperatures) and therefore the observed high frequency spectrum of the thermal speed is mainly produced by the incompressible part of the magnetic field perturbations, thus explaining its similarity with the proton velocity spectrum.

*grigol_gogoberidze@iliauni.edu.ge, Corresponding author

${ }^{\dagger}$ g.machabeli@iliauni.edu.ge

‡voitenko@oma.be 


\section{Thermal spectra from MHD to kinetic scales}

For the proton velocity distribution $f(\vec{v})$ the current $d I$ measured by the Faraday cup due to an elementary volume $d^{3} v$ in the velocity space is (Kasper et al., 2002)

$$
d I=e A f(\vec{v}) \hat{n} \cdot \vec{v} d^{3} v
$$

Here $A$ is the effective area of the Faraday cup and $\hat{n}$ is the direction along the main axes of the cylinder. The particle distribution function of protons in the solar wind is not isotropic and can be fitted by bi-Maxwelian distribution with different temperatures along and perpendicular to the mean magnetic field. The total Faraday cup current $\Delta I$ due to the bi-Maxwelian distribution can be calculated by integrating equation (1) over all proton velocities perpendicular to $\hat{n}$ and within some speed window along the line of sight. As showed by Kasper et al. (2002), the current $\Delta I$ measured by the Faraday cup depends not on the parallel $\left(T_{\|}\right)$and perpendicular $\left(T_{\perp}\right)$ temperatures, but only on their linear combination

$$
T_{n}=T_{\|}(\hat{n} \cdot \hat{b})^{2}+T_{\perp}\left[1-(\hat{n} \cdot \hat{b})^{2}\right] .
$$

Here $\hat{b}$ is the unit vector in the direction of the magnetic field.

One can derive the the proton distribution function moments (density, velocity, parallel and perpendicular temperatures) using a fitting procedure for the current measurements $\Delta I$ either of different Faraday cups (as in the case of very high resolution data of Spektr-R or of the same Faraday cups in different speed intervals (Kasper et al., 2002). From the above consideration it is clear that if the proton distribution function is assumed isotropic, then the corresponding isotropic temperature in both fitting algorithms will be given by the weighted sum of parallel and perpendicular temperatures (2). This is exactly the case with the Spectr-R data. Indeed, the algorithm of derivation of the solar wind plasma parameters with extremely high resolution (32 ms) (Safrankova et al., 2013) implies using the simultaneous measurements of 6 Faraday cups to fit five plasma parameters: three components of the particle flux, flow speed and temperature. However, this method works fine only in the case of isotropic temperature $T_{\|}=T_{\perp}$. In the case of anisotropic temperature, $T_{\|}$and $T_{\perp}$ separately cannot by found, only their linear combination $T_{n}$ is accessible.

Let us introduce the angle $\theta$ between the total magnetic field $\mathbf{B}$ and $\hat{n}$, such that the measured temperature $T_{n}$ (2) can be presented as (Gogoberidze et al., 2013, 2018)

$$
T_{n}=\left(T_{\|}-T_{\perp}\right) \cos ^{2} \theta+T_{\perp} .
$$

Furthermore, we will distinguish the mean and fluctuating parts of anisotropic temperatures $T_{\|, \perp}$ and magnetic field $\mathbf{B}$ :

$$
T_{\|}=T_{0 \|}+\delta T_{\|} ; \quad T_{\perp}=T_{0 \perp}+\delta T_{\perp} ; \quad \mathbf{B}=\mathbf{B}_{0}+\delta \mathbf{B}=\mathbf{B}_{0}+\delta \mathbf{B}_{\|}+\delta \mathbf{B}_{\perp}
$$

Then $\cos \theta \operatorname{can}$ be expressed in terms of fluctuating magnetic fields exactly,

$$
\cos \theta=\sqrt{1-\left(\frac{\delta B_{\perp}}{B}\right)^{2}} \cos \theta_{0}+\frac{\delta B_{\perp}}{B} \cos \phi \sin \theta_{0},
$$

where $\theta_{0}$ is the angle between the mean magnetic field $\mathbf{B}_{0}$ and $\hat{n}, \phi$ is the angle between $\delta \mathbf{B}_{\perp}$ and $\left(\hat{n}, \mathbf{B}_{0}\right)$ plane, and $B=|\mathbf{B}|$,

$$
B=\sqrt{B_{0}^{2}+2 B_{0} \delta B_{\|}+\left(\delta B_{\|}\right)^{2}+\left(\delta B_{\perp}\right)^{2}} .
$$

Note that $\cos \theta$ does not depend on $\delta B_{\|}$if $\delta B_{\perp}=0$.

In general, as follows from (3-5), fluctuations of $T_{n}$ are caused by the fluctuations of all involved parameters: $\delta T_{\|}, \delta T_{\perp}, \delta B_{\|}$, and $\delta B_{\perp}$. Therefore, the measured spectrum of $T_{n}$, or thermal velocity $\sim \sqrt{T_{n}}$ used by Safrankova et al. (2013), contains contributions of all these sources. 
Temperature spectrum of the solar wind turbulence

In what follows we show that, for the typical solar wind parameters, the dominant contribution to the spectra of $T_{n}$ and $\sqrt{T_{n}}$ comes from the magnetic fluctuations rather than the fluctuations of parallel and perpendicular temperatures. First, we present

$$
\cos ^{2} \theta=\cos ^{2} \theta_{0}+\delta\left(\cos ^{2} \theta\right),
$$

where the $\delta \mathbf{B}$-dependent fluctuating part

$$
\delta\left(\cos ^{2} \theta\right)=\left(\left(\cos \phi \sin \theta_{0}\right)^{2}-\cos ^{2} \theta_{0}\right)\left(\frac{\delta B_{\perp}}{B}\right)^{2}+\sin \left(2 \theta_{0}\right) \cos \phi \sqrt{1-\left(\frac{\delta B_{\perp}}{B}\right)^{2}} \frac{\delta B_{\perp}}{B}
$$

All above expressions (3-8) are quite general, valid for any values of fluctuating parameters. To simplify further analysis we consider the limit of small perturbations, $\delta B_{\perp} / B, \delta T / T \ll 1$. Although this condition is not always satisfied for low frequency magnetic field perturbations it is always valid for the high frequency perturbations close to the spectral break between MHD and kinetic ranges. Then, using (8) in (3) and retaining only leading terms with respect to the small parameters $\delta B_{\perp} / B, \delta T / T \ll 1$, the fluctuating part of can be simplified to

$$
\frac{\delta T_{n}}{T_{0 \perp}}=\cos ^{2} \theta_{0} \frac{\delta T_{\|}}{T_{0 \perp}}+\sin ^{2} \theta_{0} \frac{\delta T_{\perp}}{T_{0 \perp}}+\left(\frac{T_{0 \|}}{T_{0 \perp}}-1\right) \sin \left(2 \theta_{0}\right) \cos \phi \frac{\delta B_{\perp}}{B_{0}} .
$$

The dimensionless spectral power of $T_{n}$ perturbations is thus

$$
\frac{\left\langle\delta T_{n}^{2}\right\rangle}{T_{0 \perp}^{2}}=\frac{\left\langle\delta T_{n}^{2}\right\rangle_{T}}{T_{0 \perp}^{2}}+\frac{\left\langle\delta T_{n}^{2}\right\rangle_{B}}{T_{0 \perp}^{2}}
$$

where we introduce the thermal contribution

$$
\frac{\left\langle\delta T_{n}^{2}\right\rangle_{T}}{T_{0 \perp}^{2}}=\left\langle\left(\cos ^{2} \theta_{0} \frac{\delta T_{\|}}{T_{0 \perp}}+\sin ^{2} \theta_{0} \frac{\delta T_{\perp}}{T_{0 \perp}}\right)^{2}\right\rangle
$$

and the pseudo-thermal contribution due to magnetic fluctuations

$$
\frac{\left\langle\delta T_{n}^{2}\right\rangle_{B}}{T_{0 \perp}^{2}}=\frac{1}{2}\left(\frac{T_{0 \|}}{T_{0 \perp}}-1\right)^{2} \sin ^{2}\left(2 \theta_{0}\right) \frac{\left\langle\delta B_{\perp}^{2}\right\rangle}{B_{0}^{2}} .
$$

Here $\langle\ldots\rangle$ is the ensemble average and we assume that the turbulence is symmetric with respect to $\phi$ : $\langle\cos \phi\rangle=0 ;\left\langle\cos ^{2} \phi\right\rangle=1 / 2$.

The spectral power density of the "thermal velocity" $V_{T n}=\sqrt{V_{T \|}^{2} \cos ^{2} \theta+V_{T \perp}^{2} \sin ^{2} \theta}$ can be found similarly. Again, it consists of two parts, thermal

$$
\frac{\left\langle\left|\delta V_{T n}\right|^{2}\right\rangle_{T}}{V_{T 0 \perp}^{2}}=\frac{1}{4} \frac{\cos ^{2} \theta_{0} \delta\left(V_{T \|}^{2}\right)+\sin ^{2} \theta_{0} \delta\left(V_{T \perp}^{2}\right)}{V_{T 0 \perp}^{2}}
$$

and pseudo-thermal (due to magnetic fluctuations)

$$
\frac{\left\langle\left|\delta V_{T n}\right|^{2}\right\rangle_{B}}{V_{T 0 \perp}^{2}}=\frac{1}{8} \frac{\left(T_{0 \|} / T_{0 \perp}-1\right)^{2} \sin ^{2}\left(2 \theta_{0}\right)}{\left(T_{0 \|} / T_{0 \perp}-1\right) \cos ^{2} \theta_{0}+1} \frac{\left\langle\delta B_{\perp}^{2}\right\rangle}{B_{0}^{2}} .
$$

It is long known that in the inertial interval of the solar wind turbulence the dimensionless magnetic fluctuations $\delta B_{\perp} / B_{0}$ associated with incompressible Alfvén waves are about one order of magnitude higher than the amplitudes of compressional fluctuations associated with perturbations of density and/or temperature, $\sim \delta T_{\|, \perp} / T_{0 \perp}$ (Gogoberidze et al., 2018):

$$
\frac{\delta B_{\perp}}{B_{0}} \gg \frac{\delta T_{\|}}{T_{0 \perp}}, \frac{\delta T_{\perp}}{T_{0 \perp}}
$$


Then from (12) it is seen that, excluding specific cases when $\sin 2 \theta_{0} \leq 0.1$ or $\left|T_{0 \|} / T_{0 \perp}-1\right| \leq 0.1$, the measured power spectra of $T_{n}$ (as well as the corresponding power spectra of $V_{T n}$ ) are dominated by the Alfvénic magnetic fluctuations rather than the fluctuations of the temperature itself.

To estimate the maximum of the thermal contribution (the first term in the rhs of equation (10)) we assume that at any timescale $\tau$ the perturbations $\delta T_{\perp}(\tau)$ and $\delta T_{\|}(\tau)$ are equal $\delta T_{\perp}=\delta T_{\|}=\delta T$ and perfectly correlated. In this case after averaging the thermal contribution in the dimensionless rms power of perturbations is

$$
\frac{\left\langle\delta T_{n}^{2}\right\rangle_{T}}{T_{0 \perp}^{2}} \approx \frac{\left\langle\delta T^{2}\right\rangle}{T_{0 \perp}^{2}} .
$$

Now we estimate the magnetic contribution to the observed spectrum. As follows from equation (12), this contribution vanishes if $T_{0 \|}=T_{0 \perp}$ or $\sin 2 \theta_{0}=0$. Although in the slow solar wind there are intervals with $T_{0 \|} \approx T_{0 \perp}$, usually difference between parallel and perpendicular temperatures is quite significant. Taking as the typical values $\sin ^{2} 2 \theta_{0} \sim\left|\frac{T_{0 \|}}{T_{0 \perp}}-1\right| \sim 0.5$ (Kasper et al., 2002), contribution of the Alfvén waves reduces to

$$
\frac{\left\langle\delta T_{n}^{2}\right\rangle_{B}}{T_{0 \perp}^{2}} \approx \frac{1}{8} \frac{\left\langle\delta B_{\perp}^{2}\right\rangle}{B_{0}^{2}} .
$$

Estimations indicate that the ratio of dimensionless rms amplitudes $\lambda=\left(\delta T^{2} / T^{2}\right) /\left(\delta B_{\perp}^{2} / B_{0}^{2}\right)$ in the inertial range of stationary solar wind sub-intervals is usually of the order $10^{-2}$ and almost never exceeds $10 \%$ even in the slow streams of the solar wind, which are known to be much more compressible compared to perturbations in the fast solar wind.

\section{Discussion and conclusions}

We have shown that the nature of "temperature fluctuations" derived from the currents of Faraday $\operatorname{cup}(\mathrm{s})$ is strongly affected by the temperature anisotropy. If the temperature anisotropy is close to zero, then the derived "temperature fluctuations" are dominated by the perturbations of real temperature (thermal speed). However, when the temperature anisotropy is finite, as is typical for the solar wind, the "temperature fluctuations" change their source and nature being driven by the Alfvénic magnetic fluctuations rather than the fluctuating parallel and/or perpendicular temperatures. Such "temperature fluctuations", presented by (12) and (14), we call pseudo-thermal. The pseudo-thermal fluctuations and the bulk speed fluctuations have the common source, Alfvén waves, which explains a close similarity of their spectra.

The pseudo-thermal spectra should be even more similar (but with different amplitudes) to the magnetic spectra with which they share the same source - Alfvénic magnetic fluctuations. Therefore, as the magnetometer onboard Spektr-R is not operational (Safrankova et al., 2013) and in situ magnetic spectra are not available, a good proxy for them can be provided by the pseudo-thermal spectra. These spectra may be useful for testing some theoretical predictions even in the absence of magnetic data. Below we summarise several preliminary results in this direction.

1. Apparent fluctuations of the proton thermal velocity and the corresponding spectra deduced from the Spektr-R data are due to the Alfvénic magnetic fluctuations rather than the temperature fluctuations. Fluctuations of the bulk velocity and their spectra, observed simultaneously by Spektr-R, are produced by the Alfvénic velocity fluctuations. These two facts, together with the Alfvénic link between velocity and magnetic fluctuations, explain why the pseudo-thermal velocity spectra are so similar to the bulk velocity spectra.

2. We argued that the authentic thermal spectrum in the solar-wind turbulence (11) should closely resemble the density spectrum shown in Fig. 1c by Safrankova et al. (2013). However, this thermal spectrum is usually obscured by the magnetic pseudo-thermal spectrum and can rarely rise above it. As follows from equation (12) careful selection of the intervals with $T_{0 \|}=T_{0 \perp}$ or $\sin 2 \theta_{0} \approx 0$ could help in extracting authentic thermal spectra from the Spektr- $R$ data and compare them with the density spectra.

3. In view of above, several previous conclusions about similarity between the thermal and bulk velocity spectra (Safrankova et al., 2013) appear to be incorrect. The reason behind these mistakes is 
Temperature spectrum of the solar wind turbulence

that the authors did not distinguish the proper thermal spectrum from the pseudo-thermal spectrum established by magnetic fluctuations. Between these two, only the latter spectrum can resemble the bulk velocity spectrum. On the contrary, the authentic thermal spectrum should resemble the density spectrum, which is still subject for future experimental verification.

\section{Acknowledgements}

This work has been supported by Shota Rustaveli National Science Foundation grant FR-18-19964.

\section{References}

Bruno R., Carbone V., 2013, Liv. Rev. Sol. Phys., 10, 2

Gogoberidze G., Chapman S. C., Hnat B., 2012a, Phys. Plasmas, 19, 102310

Gogoberidze G., Chapman S. C., Hnat B., Dunlop M. W., 2012b, MNRAS, 426, 951

Gogoberidze G., Perri S., Carbone V., 2013, ApJ, 769, 111

Gogoberidze G., Voitenko Y., Machabeli G., 2018, MNRAS, 480, 1864

Hnat B., Chapman S. C., Gogoberidze G., Wicks R. T., 2011, PRE, 84, 065401

Kasper J. C., Lazarus A. J., Gary S. P., 2002, GRL, 29, 1839

Safrankova J., Nemecek Z., Prech L., Zastenker G. L., 2013, PRL, 110, 025004

Turner A. J., Gogoberidze G., Chapman S. C., 2012, PRL, 108, 085001 